\title{
AOR
}

Selected Papers of \#AolR2018: The $19^{\text {th }}$ Annual Conference of the Association of Internet Researchers Montréal, Canada / 10-13 October 2018

\section{FROM AD HOC ISSUE PUBLICS TO DISCOURSE COMMUNITIES: A YEAR OF PUBLIC DEBATE ON TWITTER}

\author{
Ehsan Dehghan \\ Digital Media Research Centre (DMRC), Queensland University of Technology (QUT)

\section{Introduction}

This paper presents an empirical investigation of the concept of ad hoc issue publics (Bruns \& Burgess, 2015, 2011), through an analysis of a national debate over a contentious topic in the Australian public sphere. In 2016, there were calls by some conservative political figures to make changes to a section of the Racial Discrimination Act (RDA). This particular section of the Act-section 18(C)-they claimed, was ambiguous and restricted freedom of speech (Norman, 2016). The dataset collected for this study covers a year of tweets discussing the matter in the Australian Twittersphere. This study argues that while communities involved in the debate were formed a priori to the issue, Twitter's affordances act as temporary bridges between disconnected communities, enabling the flow of information between them.

\section{Methodology}

The data for this study was collected using a combination of hashtags and keywords, starting from the contextual knowledge about the issue, and snowballing to find more hashtags and keywords and collect more tweets. Focusing on the range of network structures created due to the different communicative affordances of Twitter as the medium, the goal was to have a broad and comparative view of the publics and communities involved in the debate, and the communication among them. These analyses included the co-occurrence of hashtags, use of hashtags by accounts, retweeting network, @mentions network, and sharing of URLs by accounts. Additionally, these networks were overlaid on the map of the Australian Twittersphere (Bruns, Moon, Münch, \& Sadkowsky, 2017). This enables the researcher to compare the topic-related or affordance-dependent ad hoc issue publics with discoursedependent, ideology-oriented communities formed as a result of long-standing and

Suggested Citation (APA): Dehghan, E. (2018, October 10-13). From ad hoc issue publics to discourse communities: A year of public debate on Twitter. Paper presented at AolR 2018: The $19^{\text {th }}$ Annual Conference of the Association of Internet Researchers. Montréal, Canada: AolR. Retrieved from http://spir.aoir.org. 
more stable follower/followee relations among users. The study, therefore, helps empirically theorise the dynamics of communication and public debate on Twitter.

\section{Initial Findings}

The different communities created as a result of the various communicative affordances of Twitter could potentially create different network structures. In the case of this study, however, the retweet, @mention, account-domain and account-hashtag networks all take very similar forms. In all the networks, four main communities of users are present in the discourse, with one community, in particular, having more inward communicative behaviour, and being less connected to the rest of the network. A qualitative investigation of the Twitter profiles of accounts in each cluster shows that the four major communities are users interested in politics in general, progressive politics accounts, indigenous rights activists, and conservative/hard-right politics accounts. In all four networks, the conservative/hard-right cluster is more insular, in that more retweeting or @mentioning happens exclusively among the accounts in this cluster than with any other, and the use of unique hashtags or information sources is more likely to happen in this cluster.

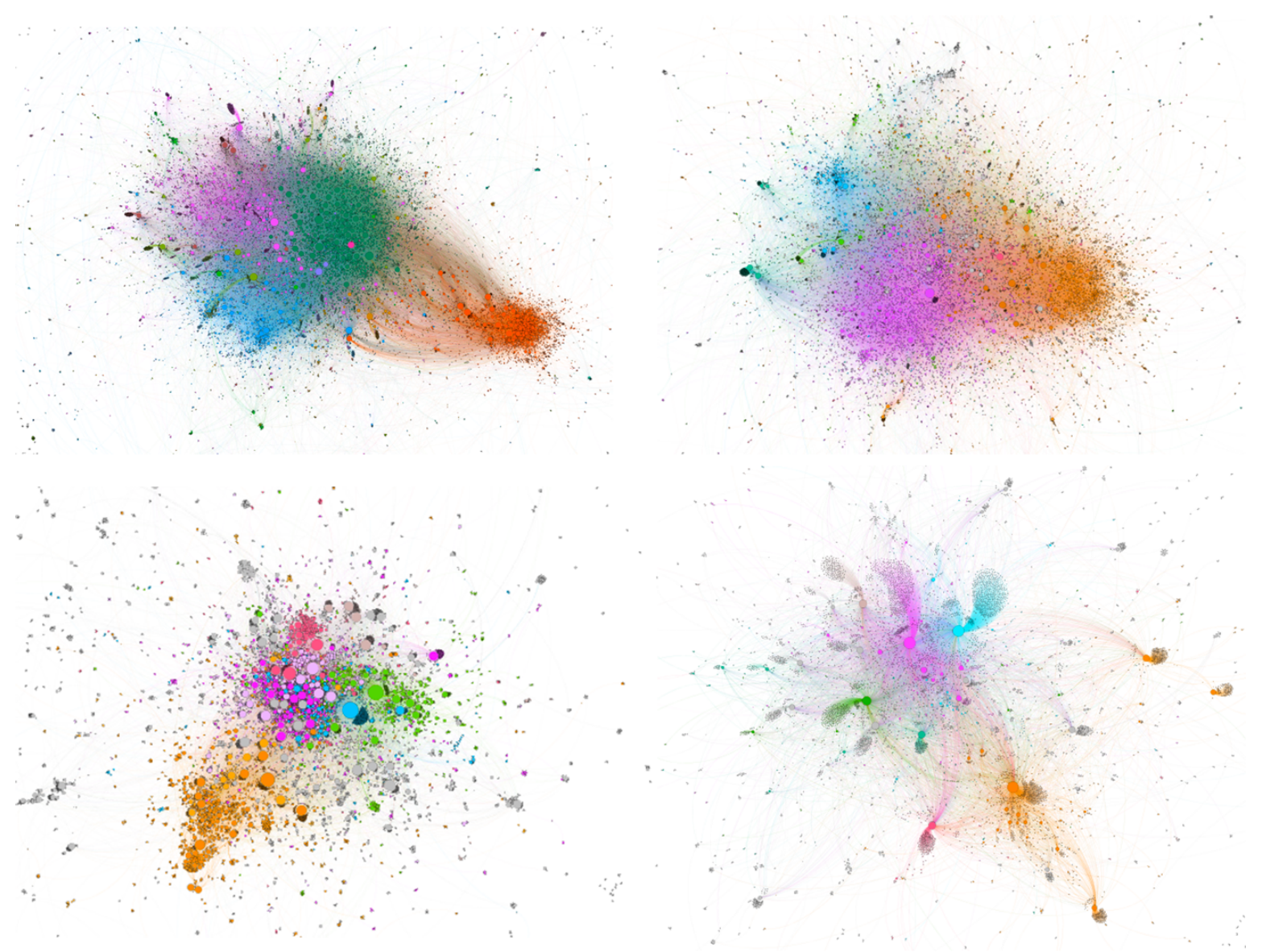

Figure 1: Top-left: Retweet network; Top-right: @mention network; Bottom-left: Account-hashtag network; Bottom-right: Account-URL network. Orange: Conservative/hard-right politics; Fuchsia: Progressive politics; Blue: Indigenous rights activists; Green: General politics 
These network structures can give a picture of the different actors and networked publics (Papacharissi \& de Fatima Oliveira, 2012) present in the debate. However, it is worth comparing these topic-related, ad hoc issue publics with more long-standing discourse communities, in order to have a clearer picture of the underlying structures that can influence the formation of ad hoc publics. To do so, the networks formed between accounts in the dataset, i.e. retweet and @mention networks, were overlaid on the map of the Australian Twittersphere. This allows examination of the interrelationship between transient, issue-related ad hoc publics with more permanent, affinity-based follower/followee relations.
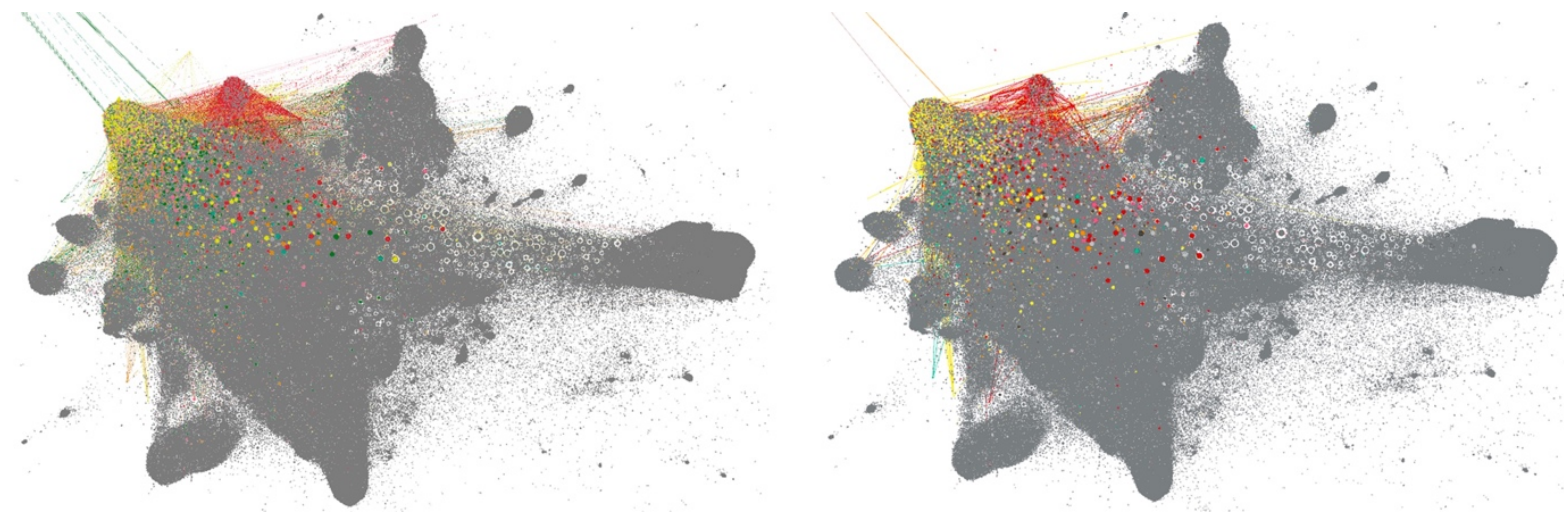

Figure 2: Overlaying the retweets and @mentions networks on the map of the Australian Twittersphere. Left: retweet; Right: @mentions; Red: accounts identified as hard-right and conservative in the dataset; Yellow: accounts identified as progressive in the dataset; Green = accounts identified as general politics in the dataset

Interestingly, the structures of the ad hoc publics closely reflect the affinity-based clusters of the Australian Twittersphere, in that the accounts identified as conservative or progressive based on the network formations in the dataset closely overlap with the network clusters formed based on follower/followee relations. In other words, although it is theoretically possible that an ad hoc issue public is created where users with a wide range of interests tweet about the issue, such is not the case here. Rather, only users who have already formed discourse communities based on their political ideologies have tweeted about the issue. In doing so, these users retweet, @mention, incorporate hashtags, or share information purely based on their ideological affinity. This raises questions regarding the suitability of the concept of ad hoc publics to refer to this debate.

\section{Discussion}

The dataset in this study represents a collection of tweets discussing a contentious topic in the Australian public sphere. This can be theorised as an ad hoc issue public, created as a result of the use of certain hashtags or keywords (e.g. \#18c and \#rda). However, digging deeper in the data reveals that the concept of an ad hoc issue public cannot satisfactorily explain the dynamics of this discursive environment. Discourse analysis of the tweets posted in each cluster shows a range of discourses at play; although all the tweets in the dataset discuss the same issue, the argumentation schemes, sources of information, communicative actions, and discourse topics are different. Therefore, it 
seems better to conceptualise this discursive environment as a collection of publics or discourse communities, rather than as a singular public.

Moreover, the publics formed around this discussion can be argued to be not ad or post $h o c$, but formed a priori to the issue or discussion, as a result of affinity-based connections among individuals; the accounts participating in the discussion enter the conversations with already formed discursive positions and ideologies, and in discussing the issue, they draw from these ideologies. The imagined audience, therefore, is different for each community in this environment. While there are common hashtags central to the discussion, it is hard to argue that these hashtags are used to talk to an imaginary audience consisting of everyone interested in the issue. Rather, seen in combination with all the other forms of communicative affordances employed on Twitter, such as @mentions, retweets, and sharing of links, the evidence suggests that the imaginary audience for each public is mostly comprised of people with the same ideologies.

In this case, therefore, the significance of Twitter's affordances lies in the fact that despite disconnected ideologies of the a priori communities, these affordances serve as temporary hybrids between long-term follower relations and short-term publics formed around the topic. That is, @mentions, retweets, and hashtags act as bridges, however temporary, between otherwise disconnected and/or antagonistic communities. Of course, this is not to argue that discourse communities and ad hoc issue publics are mutually exclusive. Rather, by using a limited number of hashtags, which form an ad hoc issue public through mere collective attention, discourse communities find a common space to perform discursive struggles and challenge each other's ideologies.

These findings also raise questions regarding the recent debates over algorithmic filter bubbles and presence of echo chambers in online discussions. To answer these questions, the next steps in this project are to investigate the links between these antagonistic discourse communities, in order to track the flow of information between them, conduct an in-depth analysis of their discursive strategies in light of the sociopolitical context, and compare the underlying dynamics of the various network structures created by the affordances of the platform. This provides new insights into the impact of algorithmic structures of the platforms and their affordances on online public debate and information flows, which in turn can be utilised to foster more democratic and less fragmented online discourses.

\section{References}

Bruns, A., \& Burgess, J. (2015). Twitter hashtags from ad hoc to calculated publics. Hashtag Publics: The Power and Politics of Discursive Networks, 13-28

Bruns, A., \& Burgess, J. E. (2011). The use of Twitter hashtags in the formation of ad hoc publics. In Proceedings of the 6th European Consortium for Political Research (ECPR) General Conference 2011

Bruns, A., Moon, B., Münch, F., \& Sadkowsky, T. (2017). The Australian Twittersphere in 2016: Mapping the Follower/Followee Network. Social Media+ Society, 3(4) 
Norman, J. (2016, August 30). The history of the Racial Discrimination Act. Retrieved February 26, 2018, from http://www.abc.net.au/news/2016-08-30/racialdiscrimination-act-explainer/7798546

Papacharissi, Z., \& de Fatima Oliveira, M. (2012). Affective News and Networked Publics: The Rhythms of News Storytelling on \#Egypt. Journal of Communication, 62(2), 266-282 